\title{
NEW STOPPING CRITERIA FOR ITERATIVE BLIND IMAGE DEBLURRING BASED ON RESIDUAL WHITENESS MEASURES
}

\author{
Mariana S. C. Almeida and Mário A. T. Figueiredo \\ Instituto de Telecomunicações and \\ Instituto Superior Técnico, Technical University of Lisbon, Portugal \\ Email: $\{$ mariana.almeida, mario.figueiredo\}elx.it.pt
}

\begin{abstract}
Blind image deblurring (BID) is a challenging ill-posed inverse problem. Most BID methods work by imposing some regularization on the unknown blur, and use iterative optimization schemes. Recently, a method was proposed that, although not requiring prior knowledge on the blurring filter, achieves state-of-the-art performance on a wide class of realworld BID problems. The drawback of that method is that the iterations have to be manually stopped.

In this paper, we propose new stopping criteria for iterative BID algorithms. The rationale behind the proposed criteria is that if the blur filter is well estimated, the residual will be spectrally white, whereas with a wrong filter, the image estimate will exhibit structured artifacts, which are not white. Comprehensive experiments using the proposed criteria to stop the method mentioned in the previous paragraph show that it yields, on average, an ISNR only $0.16 \mathrm{~dB}(\sim 3 \%)$ below what is obtained by stopping the algorithm at the best ISNR (which, of course, can't be done in practice).
\end{abstract}

Index Terms - Blind image deblurring, whiteness, stopping criteria, image deconvolution.

\section{INTRODUCTION}

Image deblurring (ID) is an inverse problem in which the observed image, $y$, is modeled as the degradation of a sharp image, $x$, by a convolution with a blurring filter $h$, plus some noise, $n$. With application in many areas (e.g., astronomy, photography, surveillance, remote sensing), fundamental research on ID can be divided into non-blind problems, in which the blurring filter $h$ is assumed to be know, and blind ID (BID), in which both the image $x$ and the blurring filter $h$ are unknown. Despite its narrower applicability, non-blind ID is already a challenging problem to which a large amount of research has been devoted, mainly due to the fact that the convolution operators of interest are typically very ill-posed.

This work was partially supported Fundação para a Ciência e Tecnologia, under grant PTDC/EEA-TEL/104515/2008 and the fellowship SFRH/BPD/69344/2010.
In BID, even if the degradation operator was not ill-posed, the problem would still be inherently ill-posed, since there is an infinite number of solutions (pairs of image estimates and blurring filters) that are compatible with the observed degraded image. In order to obtain reasonable results, almost all BID methods restrict the class of the blurring filters. These restrictions have been applied either in a hard way, through the use of parametric models $[1,2]$, or in a soft way, through the use of priors/regularizers $[3,4,5,6]$. In contrast to those approaches, a recent BID method [7] does not use prior knowledge about the blurring filter, but still achieves state-of-the-art performance on a wide range of BID problems (both synthetic and real data). That method is iterative and starts by estimating the main features (edges) of the image, by setting the regularizing parameter to a high value, and gradually learns the image and filter details, by slowly decreasing the value of the regularizing parameter. The drawback of the method is that it needs to be manually stooped, which corresponds to manually tuning the regularization parameter. In fact, finding robust stopping criteria and/or adjusting the regularization parameter in iterative (blind or non-blind) ID algorithms is a long standing problem, which is still an open research topic $[8,9,10]$.

While strategies for automatically estimating the value of the regularization parameter are relatively developed for nonblind ID $[8,9,10]$, the same is not true for the blind case. Most of the existing BID methods require the regularizing parameters to be somehow tuned or empirically selected. Despite that, there are some recent BID approaches $[3,5,6]$ that manage to determine the regularization parameter. Some of these approaches $[3,5]$ were developed for variational methods and do not fit the BID technique of [7]. The maximum a posteriori (MAP) approach from [6] did not show to work successfully in combination with the method of [7].

This paper proposes stopping criteria based on the assumption that the additive noise is spectrally white. The rationale is that if the blurring filter is correctly estimated, the residual associated to the deblurred image is white. This rationale is implemented by using measures of whiteness to assess the fitness of the current estimates to the degradation 
model. The best stopping criterion based on whiteness measures was successfully tested on a large set of experiments, leading to an average decrease of $3 \%$ of the ISNR compared to what is obtained by stopping the algorithm at the maximum ISNR (something that, of course, cannot be done in practice, as it requires the original image). Whiteness measures of the residual have been previously used to assess model accuracy $[11,12]$; however, those works are on very different areas.

\section{THE BLIND DECONVOLUTION METHOD}

The degradation we aim at inverting is modeled by

$$
y=h * x+n,
$$

where $y, x$ and $n$ are, respectively, the degraded image, the (unknown) original image, and additive noise; $h$ is the (unknown) point spread function (PSF) of the blurring operator, and $*$ denotes two-dimensional convolution.

In the BID method proposed in $[13,7]$, the pair $(h, x)$ is estimated by minimizing a cost function:

$$
C_{\lambda}(x, h)=\frac{1}{2}\|y-h * x\|_{2}^{2}+\lambda R[f(x)] .
$$

The first term in (2) is the classical data fidelity term that results from assuming that the noise $n$ is white and Gaussian, $R[f(x)]$ is a regularizing function which favors solutions in which the image edges (extracted by a function $f$ ) are sparse, and $\lambda$ is the regularizing parameter. More details on the edge extractor $f(\cdot)$ and on the regularization criterion $R($.$) can be$ found in $[13,7]$.

Among the infinite number of possible solutions $(x, h)$ for the inverse problem in (1), a suitable one is reached iteratively by starting with a strong regularization (setting $\lambda$ to a high value), which is gradually decreased (see Algorithm 1). Initial estimates consist in cartoon-like images, strongly piece-wise-constant. The sharp edges of these images, when compared with the blurred image $y$, allow to learn and improve the estimate of the blurring filter $h$, which, on the other hand, allows to reduce the strength of the regularization and to learn finer image details. This slow decrease of the regularizing parameter was shown to lead to good deblurring solutions, without imposing prior information on the blurring filter (for more details, see [7]).

So far, the stopping criterion of the method, which corresponds to setting the final value of the regularizing parameter, was manually tuned for each experiment. This was done either based on the ISNR value, in the case synthetic experiments, or by visual assessment of the restored image (in the case of real blurred images) [7]. In the following section, we describe a new method for automatically stopping this BID algorithm (or other BID algorithms).

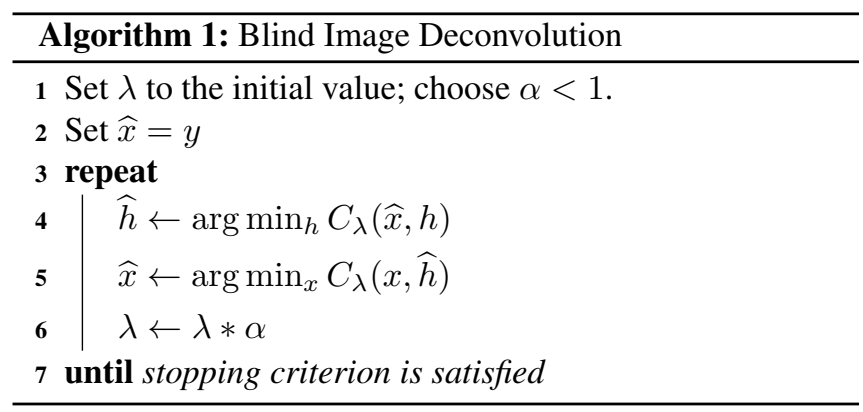

\section{THE STOPPING CRITERIA}

The proposed stopping criteria are based on measures of the fitness of the estimated data $(\widehat{x}, \widehat{h})$ to the degradation model (1), naturally based on the residual image:

$$
r=y-\widehat{h} * \widehat{x} .
$$

The statistics of the residuals $r$ are then compared with those assumed for the noise $n$ of the degradation model (1). In particular, the noise $n$ is assumed to be white (uncorrelated), thus a measure of the whiteness of the residuals $r$ is used to assess the adequacy of the estimates $(\widehat{x}, \widehat{h})$ to the model. Note that this is a relatively generic assumption, which is true for most real-life situation.

\subsection{Measures of whiteness}

The first step of our method is to normalize the residual image to zero mean and unit variance; for simplicity of notation, let this normalized residual still be denoted as $r$. The autocorrelation (and auto-variance, since the mean is zero) of the normalized residual $r$, at $2 \mathrm{D}$ lag $(m, n)$, is estimated by

$$
R_{r r}(m, n)=\sum_{i, j} r(i, j) r(i-m, j-n),
$$

where the sum extends over the whole image.

The auto-covariance of a spectrally white image is a delta function at the origin, $\delta(m, n)$. A possible measure of whiteness of $r$ is thus the distance between the estimated autocorrelation $R_{r r}(m, n)$ and the ideal delta function. Considering a window of $(2 L+1) \times(2 L+1)$ pixels, the first measure of whiteness that we consider is simply the energy of $R_{r r}(m, n)$ outside the origin,

$$
M_{R}(r)=-\sum_{\substack{m, n)=(-L,-L) \\(m, n) \neq(0,0)}}^{L, L}\left(R_{r r}(m, n)\right)^{2}
$$

where the minus sign is used so that $M_{R}(r)$ is larger for whiter residuals. In the experiments reported below, we have used $L=4$. 
Considering that the auto-covariance has more significant values for smaller lags, it makes sense to give more weight to these terms. Based on that, a weighted version of the measure in (5) was also considered,

$$
M_{R W}(r)=-\sum_{\substack{(m, n)=(-L,-L) \\(m, n) \neq(0,0)}}^{L, L} W(m, n)\left(R_{r r}(m, n)\right)^{2},
$$

where $W(m, n)$ is a matrix of weights. In the experiments, we have used a Gaussian weighting.

Let $S_{r r}\left(\omega_{1}, \omega_{2}\right)$ denote the power spectral density of $r$, at 2D spatial frequency $\left(\omega_{1}, \omega_{2}\right)$,

$$
S_{r r}\left(\omega_{1}, \omega_{2}\right)=\mathcal{F}\left(R_{r r}\right)
$$

where $\mathcal{F}$ is the two-dimensional discrete Fourier transform (2D-DFT). In agreement with the fact that the autocorrelation of a white signal is a delta function, a white signal has a flat power spectral density. To measure the flatness of $S_{r r}$, we measure its Shannon entropy, after adequate normalization; recall that the maximum entropy is achieved by a flat distribution. The resulting measure of whiteness is denoted as $M_{H}(r)$.

The stopping criterion consists in stopping the BID algorithm when the whiteness measure used $\left(M_{R}(r), M_{R W}(r)\right.$, or $\left.M_{H}(r)\right)$ starts decreasing. To avoid a premature stopping (of course, we have no guarantee that the whiteness measure does not oscillate), we actually run the algorithm until the whiteness measure decreases considerably, and then return the image estimate obtained at the iteration at which the maximum whiteness was observed.

\subsection{Local Measures of Whiteness}

The approach described in the previous subsection implicitly assumes that the residual $r$ is stationary and ergodic; i.e., we are estimating the auto-covariance by averaging over the whole image (see (4)). Of course, in practice, the residual is not stationary, in fact exhibiting clear spatial variability. These observation lead us to considered also local versions of the previous measures of whiteness, where local autocovariance estimates:

$$
R_{r r}^{b}(m, n)=\sum_{i, j \in B} r(i, j) r(i-m, j-n),
$$

where $b$ indexes an image block, and $B$ is the set of pixels in that block. In the experiments reported below, we have used $9 \times 9$ blocks, separated horizontally and vertically by 5 pixels. Of course, in this case, the residual is normalized to zero mean and unit variance on a block-by-block fashion, rather than globally. Given this block partition, the three local measures of whiteness, $M_{R}^{l}(),. M_{R W}^{l}($.$) and M_{H}^{l}($.$) were$ computed, by averaging the block-wise application of $M_{R}($.$) ,$ $M_{R W}($.$) , and M_{H}($.$) , respectively.$

\section{EXPERIMENTS}

The proposed stopping criteria were tested on most of the experiments described in [7]; specifically, we have considered: (a) four benchmark images: "Lena", "Barbara", "Cameraman" and "Satellite"; (b) the different blurring filters used in [7], out-of-focus, linear motion blur, square blur, random blur, nonlinear motion blur, and Gaussian blur; (c) experiments with no added noise and with Gaussian white noise, at a blurred-signal-to-noise-ratio (BSNRs) of 30dB; (d) experiments with and without constrains (parametric form and symmetry) on the blurring filter (see [7] for full details).

For all the possible combinations of images, blurs, and constraints descried in the previous paragraph (a total of more than 100 different experiments), the three global and local measures described in Section 3 were computed. Table 1 summarizes the results obtained using the global stopping criteria. It is clear that all three criteria (based on $M_{R}, M_{R W}$, and $M_{H}$ ) are able to stop the algorithm at estimates which are, on average, only slightly worse $(-0.39 \mathrm{~dB},-0.40 \mathrm{~dB},-0.38 \mathrm{~dB})$ than the best ISNR achieved by manual stopping. This result is also reasonably stable, as show by the standard deviation of the ISNR decrease, which is clearly below $1 \mathrm{~dB}$. This can be considered as a successful result, considering the wide set of degradations that were considered and the well-known difficulty of the BID problem.

\begin{tabular}{|c||c|c|c|c|}
\hline & $\begin{array}{c}\text { Best } \\
\text { ISNR }\end{array}$ & $\begin{array}{c}M_{H} \\
\Delta \text { ISNR }\end{array}$ & $\begin{array}{c}M_{R} \\
\Delta \text { ISNR }\end{array}$ & $\begin{array}{c}M_{R W} \\
\Delta \text { ISNR }\end{array}$ \\
\hline \hline Mean & 5,88 & $-0,39$ & $-0,40$ & $-0,38$ \\
\hline Standard dev. & 2,63 & 0,65 & 0,70 & 0,72 \\
\hline
\end{tabular}

Table 1. Experimental results (in $\mathrm{dB}$ ). First column: best ISNR obtained during the iterations. Second, third, and fourth columns: $\triangle$ ISNR denotes the differences between the ISNR obtained by stopping with each of the automatic criteria and the best ISNR.

Despite the good performance of the global whiteness measures, the local measures (Subsection 3.2) turned out to yield even better results. Table 2 shows detailed results obtained with the best local measure: $M_{R W}^{l}$. The average difference in ISNR with respect to the maximum is $-0.16 \mathrm{~dB}$, with a standard deviation of $0.21 \mathrm{~dB}$. The rightmost column of Table 2 shows the difference in number of iterations between the automatically stopped algorithm and the manually stopped version (highest ISNR), showing that the automatic criterion tends to stop slightly earlier. In fact, only the noisy case (third row) is meaningful; in the noiseless experiments, the ISNR stabilizes in the latter iterations, thus these large differences correspond in fact to quite similar image estimates. 


\begin{tabular}{|c|c|c|c|c|}
\hline & $\begin{array}{c}\text { Best } \\
\text { ISNR }\end{array}$ & $\begin{array}{c}\Delta \text { ISNR } \\
\text { mean }\end{array}$ & $\begin{array}{c}\Delta \text { ISNR } \\
\text { st. dev. }\end{array}$ & $\begin{array}{c}\Delta \text { Iter. } \\
\text { mean }\end{array}$ \\
\hline All & $5,88 \mathrm{~dB}$ & $-0,16 \mathrm{~dB}$ & $0,21 \mathrm{~dB}$ & $-5,4$ \\
\hline noiseless & $6,90 \mathrm{~dB}$ & $-0,15 \mathrm{~dB}$ & $0,26 \mathrm{~dB}$ & $-9,9$ \\
\hline noisy & $4,85 \mathrm{~dB}$ & $-0,16 \mathrm{~dB}$ & $0,16 \mathrm{~dB}$ & $-0,9$ \\
\hline constrained & $6,60 \mathrm{~dB}$ & $-0,20 \mathrm{~dB}$ & $0,27 \mathrm{~dB}$ & $-6,7$ \\
\hline unconstrained & $5,36 \mathrm{~dB}$ & $-0,13 \mathrm{~dB}$ & $0,16 \mathrm{~dB}$ & $-4,5$ \\
\hline
\end{tabular}

Table 2. Results obtained using $M_{R W}^{l}$. First column: ISNR of the best iteration. Second and third columns: mean and standard deviation of $\triangle I S N R$ (defined as in Table 1). Third column: standard deviation of the ISNR error. Fourth column: difference in iterations.

\subsection{Real Blurred Photos}

The stopping criterion based on $M_{R W}^{l}$ was also tested on a real blurred photo (ou-of-focus). We have used one of the photos from [7] (see Fig.1, left). The proposed automatic criterion stopped the algorithm at an iteration in which the estimated image (Fig. 1, right) is equal to the best estimate that was visually obtained along the algorithm.

\section{CONCLUSIONS AND FUTURE WORK}

We have proposed stopping criteria for iterative blind image deconvolution algorithms, based on whiteness measures applied to the residual error. We have used the proposed stopping criteria with a recent state-of-the-art method; on a wide range of synthetic experiments, we showed that the best of the proposed criteria yields ISNR losses with the respect to the best ISNR of only $0.16 \mathrm{~dB}$, on average. The method was also successfully tested on a real blurred photo, although more experiments on real data are needed and are under way.

The proposed approach is quite general and does not require any knowledge about the type of convolution operator. This fact makes the proposed criteria easily applicable to other blind or non-blind deconvolution methods, a research direction that we plan to explore in the near future.

\section{REFERENCES}

[1] J. P. A. Oliveira, M. A. T. Figueiredo, J. M. Bioucas-Dias, "Blind estimation of motion blur parameters for image deconvolution,” in IbPRIA, Spain, 2007.

[2] M. S. Chang, S. W. Yun, P. Park, "PSF search algorithm for dual-exposure type blurred image," Intern. Jour. Appl. Science, Engineering and Technology, vol. 4, 2007.

[3] R. Fergus, B. Singh, A. Hertzmann, S. T. Roweis, W. T. Freeman, "Removing camera shake from a single photograph," ACM Trans. Graphics, vol. 25, pp. 787-794, 2006.

[4] Q. Shan, J. Jia, A. Agarwala, "High-quality motion deblurring from a single image," ACM Trans. Graphics, vol. 27, 2008.
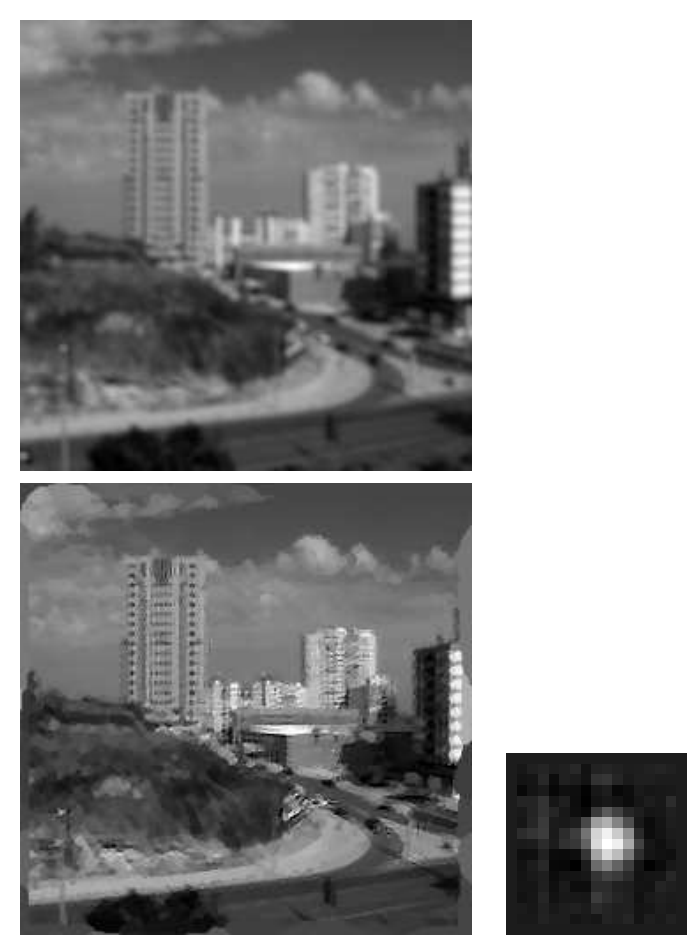

Fig. 1. Results on a real blurred photo. Top: the blurred image. Bottom: the restored image and the estimated filter.

[5] S. D. Babacan, R. Molina, A. K. Katsaggelos, "Variational Bayesian blind deconvolution using a total variation prior," IEEE Trans. Image Processing, vol. 18, pp. 12-26, 2009.

[6] B. Amizic, S. D. Babacan, R. Molina, A. K. Katsaggelos, "Sparse Bayesian blind image deconvolution with parameter estimation," Proc. EUSIPCO, 2010.

[7] M. S. C. Almeida, L. B. Almeida, "Blind and semi-blind deblurring of natural images," IEEE Trans. Image Processing, vol. 19, pp. 36-52, 2010.

[8] J. P. Oliveira, J. M. Bioucas-Dias, M. A. T. Figueiredo, "Adaptive total variation image deblurring: A majorizationminimization approach," Signal Processing, vol. 89, pp. 16831693, 2009.

[9] G. K. Chantas, N. P. Galatsanos, R. Molina, A. K. Katsaggelos, "Variational Bayesian image restoration with a product of spatially weighted total variation image priors," IEEE Trans. Image Processing, vol. 19, pp. 351-362, 2010.

[10] R. Giryes, M. Elad, Y. C. Eldar, "The projected GSURE for automatic parameter tuning in iterative shrinkage methods," Appl. and Comput. Harmonic Anal., to appear, 2011.

[11] E. Djermoune, G. Kasalica, D. Brie, "Estimation of the parameters of two-dimensional NMR spectroscopy signals using an adapted subband decomposition," Proc. IEEE ICASSP, 2008.

[12] E. J. Sullivan, N. Xiang, J. V. Candy, "Adaptive model-based mine detection/localization using noisy laser Doppler vibration measurements," Proc. IEEE OCEANS, 2009.

[13] M. S. C. Almeida, L. B. Almeida, "Blind deblurring of natural images," Proc. IEEE ICASSP, 2008. 